Canadian Journal of Fisheries and Aquatic Sciences

Canadian Science Publishing

Journal canadien des sciences halieutiques et aquatiques

\title{
Detecting Regime Shifts in Fish Stock Dynamics
}

\begin{tabular}{|r|l|}
\hline Journal: & Canadian Journal of Fisheries and Aquatic Sciences \\
\hline Manuscript ID: & cjfas-2014-0406.R2 \\
\hline Manuscript Type: & Article \\
\hline Date Submitted by the Author: & 05-Jun-2015 \\
\hline Complete List of Authors: & $\begin{array}{l}\text { Perälä, Tommi; University of Helsinki, Department of Environmental } \\
\text { Sciences } \\
\text { Kuparinen, Anna; University of Helsinki, Department of Environmental } \\
\text { Sciences }\end{array}$ \\
\hline Keyword: & $\begin{array}{l}\text { BAYESIAN STATISTICS < General, PRODUCTIVITY < General, } \\
\text { RECRUITMENT < General, TIME SERIES ANALYSIS < General }\end{array}$ \\
\hline &
\end{tabular}

SCHOLARONE

Manuscripts 


\section{Detecting regime shifts in fish stock dynamics}

3 Tommi Perälä ${ }^{1 *}$, Anna Kuparinen ${ }^{1}$

4

$5{ }^{1}$ Department of Environmental Sciences, P. O. Box 65, 00014 University of Helsinki,

6 FiGlarrdsponding author:+358503185072; tommi.perala@helsinki.fi

7

8 


\section{Abstract}

10 Environmental factors such as the water temperature, salinity and the abundance of

11 zooplankton can have significant effects on certain fish stocks' ability to produce juveniles

12 and, thus, stock renewal ability. This variability in stock productivity manifests itself as

13 different productivity regimes. Here, we detect productivity regime shifts by analyzing

14 recruit-per-spawner time series with Bayesian online change point detection algorithm. The

15 algorithm infers the time since the last regime shift (change in mean or variance or both) as

16 well as the parameters of the data generating process for the current regime sequentially. We

17 demonstrate the algorithm's performance using simulated recruitment data from an

18 individual-based model, and further apply the algorithm to stock assessment estimates for four

19 Atlantic cod stocks obtained from RAM legacy data base. Our analysis shows that the

20 algorithm performs well when the variability between the regimes is high enough compared to

21 the variability within the regimes. The algorithm found several productivity regimes for all

22 four cod stocks, and the findings suggest that the stocks are currently in low productivity

23 regimes, which have started during the 1990s and 2000s.

24

25 Keywords: Atlantic cod, Gadus morhua, environmental change, productivity, recruitment, time 26 series 


\section{1. Introduction}

28 Marine ecosystems can undergo rapid changes from one relatively stable state to another due to 29 alterations in environmental conditions. These changes from one state to another are often 30 called regimes shifts (Holbrook et al. 1997; Peterson and Schwing 2003; DeYoung et al. 2004).

31 More rigorously, a regime shift can be defined as the event where some internal processes or 32 external forcing drive the state of a system over a critical threshold after which the qualitative 33 behavior of the system changes. From the modelling perspective, these shifts pose an

34 interesting problem. Models often predict the behavior of the system relatively well only 35 within a narrow range of previously observed behaviors, whereas sudden changes or regime 36 shifts can be extremely difficult to predict. There are basically two ways to cope with regime

37 shifts in models. The first is to increase the model complexity to better represent the actual

38 dynamics of the system. This, however, might prove difficult due to the non-linear and chaotic 39 nature of many natural systems, lack of direct observations of the system and high uncertainty 40 about the model structure and parameters. The second way to deal with regime shifts is to use 41 simple models but make them robust to sudden changes in the model parameters. Our present 42 study falls into the latter category. We assume that the model structure is constant and define a 43 regime shift as a sudden change in the model parameters, essentially assuming that the model 44 describes the system well locally.

45 In the fisheries context, regime shifts can affect the stock productivity and further 46 translate into changes in relative species abundances. For example in the central Baltic Sea, the 47 late 1980 s regime shift is believed to have contributed to decreased cod recruitment and thus to 48 the lack of recovery of the declined Baltic cod stock. The low abundance of cod, in turn, 49 translated into reduced predation pressure on sprat which together with favorable temperature 
50 conditions drove the sprat stock to exceptionally high abundance (Alheit et al. 2005).

51 Similarly, evidence of a regime shift affecting the organization of the fish community has been 52 found in the North Sea. The 1980s regime shift increased the recruitment of flatfish whereas

53 the recruitment of gadoids declined (Beaugrand 2004). Regime shifts in juvenile productivity

54 are likely to substantially alter fish stock resilience to fishing and its ability to recover from

55 overfishing. Therefore, detection of regime shifts is vital to adjust fisheries management to

56 altered productivity conditions. Failure to do this can lead to overfishing and increase the risk

57 of fish stock collapse. Such failure is argued to have happened in Newfoundland cod (Gadus

58 morhua) fishery in Canada in the early 1990s, which lead to the collapse of the cod stock and

59 caused massive socio-economic costs (Biggs et al. 2009). On the other hand, an undetected

60 shift to a higher productivity regime can lead to underfishing due to lower expectations of

61 potential fishery yield.

62 The study of regime shifts can be roughly divided into three main problems. The first

63 problem is to identify regime shifts in the past using historical data. This retrospective analysis

64 aims at finding out when regime shifts have happened, how frequently regime shifts occur, and

65 what is the magnitude of such shifts. The second problem is to estimate model parameters

66 under the assumption of shifting regimes. The main idea is that if conditions have changed

67 drastically, and the change has driven the system to a new regime, the old data that was

68 produced under the old conditions should not affect the new estimates. The third problem is to

69 produce predictions based on the belief about the current regime and about future regime shifts.

70 The recent attempts to study productivity regime shifts in fisheries context have been

71 using frequentist methods, namely, STARS (Rodionov 2004). For example, Vert-pre et al.

72 (2013) studied 230 fish stocks and found out that the productivity of $69.1 \%$ of the stocks was

73 best explained by a model that took regime-like productivity into account. Similarly, Wayte 
74 (2013) found a regime shift to lower productivity in south-eastern Australia jackass morwong 75 (Nemadactylus macropterus) stock that happened in 1988. STARS uses sequential Student's 76 t-test to find abrupt shifts in the mean level of the dataset, and reports the times of the regime 77 shifts as well as the mean level of data in each regime. Another approach to solving the regime 78 shift detection and parameter estimation problem is to use maximum likelihood methods.

79 Maximum likelihood estimation is able to simultaneously detect shifts in the mean and

80 variance, and it has been applied for change point detection in environmental monitoring (Liu 81 2010).

Bayesian statistics offer an attractive framework for regime shift research, since it can

83 be used to detect shifts in the mean or the variance or any other parameter of the data generating

84 process, the uncertainty about the parameter estimates can be included by the use of

85 hyperparameters in hierarchical models, and predictions are easily produced by the posterior

86 predictive distribution. There exist previous attempts to study fish productivity regime shifts

87 within Bayesian statistical framework. The regime-specific parameters for a Ricker model for

88 the Japanese sardine (Sardinops melanostictus) have been inferred using a Markov switching

89 regression model with two regimes (Munch and Kottas 2009). Their analysis shows that the

90 two-regime model outperforms a single-regime model, and they suggest that a shift to a lower

91 productivity regime happened in 1988 . The method is limited to only two regimes, although a

92 similar approach may be adopted for models with multiple regimes.

93 The Bayesian online change point detection algorithm (BOCPD; Adams and McKay

94 2007) provides computationally efficient and exact recursive inference of the most recent

95 regime shift. The algorithm updates the posterior probability distribution of the time since the

96 latest regime shift together with the posterior probability distributions of the parameters of the

97 data generating process sequentially. The number of possible regimes in the algorithm equals 
98 the number of data points, since at each time step there is a small prior probability for the start

99 of a new regime. BOCPD has been used earlier for example in satellite fault prediction (Turner 100 2010), detection of shifts between different brain states in epileptic patients (Malladi et al.

101 2013), radar tracking (Turner et al. 2013), detection of a tipping point in the amplitude of the

102 annual cycle of Arctic sea ice area (Ditlevsen 2012), detection of change points in financial

103 time series (Georgescu 2012) and bird song segmentation (Wang et al. 2013). The method has

104 not, however, been used in the fisheries context.

105 In this study, we introduce the Bayesian online change point detection algorithm into 106 the fisheries research context and utilize it to detect regime shifts in fish stock productivity by 107 applying it to recruit-per-spawner time series. The method could be also used to detect shifts in 108 recruitment, growth rates, carrying capacity, natural mortality, or any other estimated model 109 parameters that could experience sudden shifts. Because of the probabilistic nature of the

110 method, it calculates probabilities for new regimes given the input data and produces estimates 111 and predictions that include the uncertainty about the regime shifts. We examine the posterior 112 probabilities for the start of a new regime at each time step for early indication of regime shifts, 113 and use smoothing to update our knowledge about the start of the regimes in the past based on 114 the current data. We also calculate the posterior predictive distribution at each time step, which 115 could be utilized in stock projections used to manage fisheries. We demonstrate the

116 performance of the method through the model validation with simulated data, and provide 117 empirical examples for regime shift detection in recruit-per-spawner estimate time series of 118 four Atlantic cod stocks.

\section{2. Methods}

120

\subsection{Model description}


121 Bayesian online change point detection algorithm (BOCPD; Adams and McKay 2007) detects

122 sudden changes in the parameters of a data generating process. Essentially the algorithm

123 consists of sequential parameter estimations, hereafter denoted as 'runs', that are carried out in

124 parallel. At each time step a new run starts and its parameters are estimated using the data

125 obtained after the start of the run. Thus, at time $t$ we have $t$ competing runs that use different

126 amount of data to make inference about the parameters of the data generating process. The runs

127 are identified in the algorithm using their lengths, 'run length', i.e. the time since the run

128 started, or equivalently, the number of data points used to calculate the parameters of the run.

129 At each time step, the algorithm updates the parameter estimates of each run based on the latest

130 data point, and calculates the probabilities of the different runs based on their probabilities at

131 the previous time step and how well the predictive density of the given run explains the latest

132 data point. Thus, the idea is that after a regime shift a new run would estimate the parameters of

133 the new regime more accurately than any of the previously started runs, and by its better

134 predictive performance would obtain high posterior probability. The posterior predictive

135 density of the model is a weighted sum of different regime specific predictive densities and the

136 weights are the posterior probabilities of the regimes. In the following, we provide a brief

137 description of the algorithm.

139 Joint distribution of data and run length

140 The joint distrbution of data $x_{1: t}=\left(x_{1}, \ldots, x_{t}\right)$ and run length $r_{t} \in\{1, \ldots, t\}$ at time $t$ can be 141 formulated recursively (for derivation see Appendix A)

$$
p\left(r_{t}, x_{1: t}\right)=\sum_{r_{t-1}=0}^{t-1} p\left(r_{t} \mid r_{t-1}\right) p\left(x_{t} \mid x^{\left(r_{t-1}\right)}\right) p\left(r_{t-1}, x_{1: t-1}\right)
$$


142 where $p\left(r_{t} \mid r_{t-1}\right)$ is the change point prior distribution that contains our prior belief of the

143 probability of a regime shift. The underlying predictive model (UPM) $p\left(x_{t} \mid x^{\left(r_{t-1}\right)}\right)$ is the

144 predictive density conditional on run length $r_{t-1}$, i.e. the predictive density using the latest

$145 r_{t-1}$ data points $x_{\left(t-r_{t-1}\right):(t-1)}$ denoted for clarity by $x^{\left(r_{t-1}\right)}$. The UPM is defined by the data

146 generating model, and it weights the probabilities of different run lengths by how well the

147 predictive density associated with the given run predicts the new data point. If the new data

148 point is 'far away' from any of the predictive densities of the existing regimes, the prior

149 predictive density $p\left(x_{t} \mid x^{(0)}\right)$ will dominate, which serves as an indicator for a start of a new

150 regime. The last term $p\left(r_{t-1}, x_{1: t-1}\right)$ is the joint distribution of data and run length at the

151 previous time step $t-1$. The summation is carried out for all possible run lengths from 0

152 (new regime) to $t-1$ (the longest possible regime so far). For simplicity, we assume that the

153 first regime starts at the beginning of the time series, and initialize the run length distribution so

154 that $p\left(r_{0}=0\right)=1$.

155

\section{Change point prior}

157 The change point prior probabilities are defined using a constant probability of a change point

$$
p\left(r_{t} \mid r_{t-1}\right)=\left\{\begin{aligned}
\frac{1}{\lambda}, & \text { if } r_{t}=0 \\
1-\frac{1}{\lambda}, & \text { if } r_{t}=r_{t-1}+1 \\
0, & \text { otherwise }
\end{aligned}\right. \text { ， }
$$

158 which gives rise, a priori, to geometric inter-arrival times for regime shifts with expected

159 length of $\lambda>0$. The change point prior has non-zero probability only for two cases: the run

160 length grows by one, i.e., the previous regime continues, or the run length goes to zero, i.e., a

161 new regime starts. 


\section{Underlying predictive model}

164 In the present study, we assume that the data-generating process is Gaussian with unknown

165 mean and precision and place a normal-gamma prior on the parameters. The likelihood is

$$
p\left(x_{t} \mid \mu, \tau\right)=\sqrt{\frac{\tau}{2 \pi}} e^{-\frac{1}{2} \tau\left(x_{t}-\mu\right)^{2}}
$$

166 where $\mu$ denotes the mean and $\tau$ the precision (reciprocal of the variance) parameter. The

167 normal-gamma prior density has the form

$$
p(\mu, \tau)=\frac{\beta^{\alpha} \sqrt{\kappa}}{\Gamma(\alpha) \sqrt{2 \pi}} \tau^{\alpha-\frac{1}{2}} e^{-\beta \tau} e^{-\frac{1}{2} \kappa \tau(\mu-\gamma)^{2}}
$$

168 where $\gamma$ is the mean location parameter, $\kappa$ is the mean scale parameter, $\alpha$ is the precision

169 shape parameter, and $\beta$ is the precision inverse scale parameter. The normal-gamma

170 distribution is the conjugate prior for the normal likelihood, and it is particularly convenient in

171 this context since the posterior distribution of the parameters conditional on run length $r_{t}$,

$172 p\left(\mu, \tau \mid r_{t}, x_{1: t}\right)=p\left(\mu, \tau \mid x^{\left(r_{t}\right)}\right)$, becomes also a normal-gamma distribution and its

173 hyperparameters $\left\{\gamma_{t}^{\left(r_{t}\right)}, \kappa_{t}^{\left(r_{t}\right)}, \alpha_{t}^{\left(r_{t}\right)}, \beta_{t}^{\left(r_{t}\right)}\right\}$ can be updated recursively as new data arrives.

174 Moreover, these assumptions regarding the stochastic process for $x_{t}, \mu$, and $\tau$ result in a

175 UPM that is a non-standardized Student's t-distribution (Murphy 2007),

$$
p\left(x_{t+1} \mid x^{\left(r_{t}\right)}\right)=t_{v_{t}^{\left(r_{t}\right)}}\left(x_{t+1} \mid \xi_{t}^{\left(r_{t}\right)}, \omega_{t}^{\left(r_{t}\right)}\right)
$$

176 where the degrees of freedom $v_{t}^{\left(r_{t}\right)}=2 \alpha_{t}^{\left(r_{t}\right)}$, the location parameter $\xi_{t}^{\left(r_{t}\right)}=\gamma_{t}^{\left(r_{t}\right)}$, and the

177 scale parameter $\omega_{t}^{\left(r_{t}\right)}=\frac{\beta_{t}^{\left(r_{t}\right)}\left(\kappa_{t}^{\left(r_{t}\right)}+1\right)}{\alpha_{t}^{\left(r_{t}\right)} \kappa_{t}^{\left(r_{t}\right)}}$, can all be expressed in terms of the posterior 
178 hyperparameters. The probability density function of the non-standardized Student's

179 t-distribution is presented in Appendix A.

180 The hyperparameter update recursions are as follows. The mean location is a weighted

181 average of the previous mean location and the newly obtained data point $x_{t}$

$$
\gamma_{t}^{\left(r_{t}\right)}=\frac{\kappa_{t-1}^{\left(r_{t}-1\right)} \gamma_{t-1}^{\left(r_{t}-1\right)}+x_{t}}{\kappa_{t-1}^{\left(r_{t}-1\right)}+1}
$$

182 The mean scale is increased by the number of new observations $(=1)$

$$
\kappa_{t}^{\left(r_{t}\right)}=\kappa_{t-1}^{\left(r_{t}-1\right)}+1
$$

183 The precision shape is increased by one half times the number of new observations $\left(=\frac{1}{2}\right)$

$$
\alpha_{t}^{\left(r_{t}\right)}=\alpha_{t-1}^{\left(r_{t}-1\right)}+\frac{1}{2}
$$

184 The precision inverse scale is incremented by the squared difference of the previous mean

185 location and the new data point scaled by one half of the ratio of the old and new mean scale 186 parameters

$$
\beta_{t}^{\left(r_{t}\right)}=\beta_{t-1}^{\left(r_{t}-1\right)}+\frac{\kappa_{t-1}^{\left(r_{t}-1\right)}\left(x_{t}-\gamma_{t-1}^{\left(r_{t}-1\right)}\right)^{2}}{2\left(\kappa_{t-1}^{\left(r_{t}-1\right)}+1\right)}
$$

187 Here the superscript $\left(r_{t}\right)$ denotes the hyperparameters obtained by using the last $r_{t}$ data

188 points, i.e., the data belonging to the regime that started $r_{t}$ time steps ago. The hyperparameter 189 updating at each step depends only on the new data point and the previous hyperparameter 190 values of the run, and is carried out sequentially when new data point arrives. The parameters

191 are updated for every possible run length $r_{t} \in\{1,2, \ldots, t\}$, and thus each possible regime has

192 different mean and precision. The hyperparameter updating is computationally fast since it 193 requires only applying the four simple recursions $t$ times. The initial values for the recursions 
194 are the prior hyperparameters for a new regime at any time step $t,\left\{\gamma_{t}^{(0)}, \kappa_{t}^{(0)}, \alpha_{t}^{(0)}, \beta_{t}^{(0)}\right\}=$

$195\left\{\gamma^{(0)}, \kappa^{(0)}, \alpha^{(0)}, \beta^{(0)}\right\}$, which are given a priori as fixed parameters of the algorithm.

196

197 Run length posterior

198 The posterior run length probabilities, i.e. the probability of a run length conditioned on the 199 data observed so far, can be computed from the joint distribution of data and run length

$$
p\left(r_{t} \mid x_{1: t}\right)=\frac{p\left(r_{t}, x_{1: t}\right)}{\sum_{r_{t}=0}^{t} p\left(r_{t}, x_{1: t}\right)}
$$

200 The joint distribution is discrete for run length $r_{t}$ with $t$ states and, thus, the sum is cheap to 201 compute. The run length posterior probabilities can be used as an early indicator of a regime shift. If the posterior probability that the current run length is one, $p\left(r_{t}=1 \mid x_{1: t}\right)$, is high, it means that the latest data point cannot be well predicted by the predictive density of the

204 previous regime, thus suggesting that a regime shift has happened.

205

\section{Posterior predictive distribution}

207 The conditional probability distribution of a new data point at time $t+1$, denoted by $x_{t+1}$,

208 conditioned on all the previous data points $x_{1: t}$, i.e. the posterior predictive distribution may be

209 written as a weighted sum of conditional probability distributions of $x_{t+1}$ conditioned on data

210 points associated with the run length $r_{t}$, i.e. the last $r_{t}$ observations $x_{\left(t-r_{t}+1\right): t}$ denoted by

$211 x^{\left(r_{t}\right)}$ (for derivation see Appendix)

$$
p\left(x_{t+1} \mid x_{1: t}\right)=\sum_{r_{t}=0}^{t} p\left(r_{t} \mid x_{1: t}\right) p\left(x_{t+1} \mid x^{\left(r_{t}\right)}\right)
$$


212 The summation is carried out over all possible run lengths, and the weights are given by the

213 posterior probabilities of the run lengths $p\left(r_{t} \mid x_{1: t}\right)$. The posterior predictive distribution is

214 used when making predictions about the future, and it is thus an integral quantity used to

215 project stock development and produce management advice.

216

\section{Smoothed run length distribution}

218 It is possible that the latest data point is an outlier instead of the first data point in a new regime.

219 In this case the posterior run length probabilities might indicate a regime shift even though no

220 shift has actually happened. Conversely, because the posterior run length probability of a new

221 regime depends on the often relatively small prior probability of a new regime and the prior

222 predictive density of a new regime, the posterior run length probability may not react quickly

223 enough to relatively small shifts. In both cases, the next data points might carry the necessary

224 information to decide if a shift has occurred. Thus, it is of interest to also compute the

225 smoothed run length distribution at each time step $t$. The smoothed run length distribution

$226 p\left(r_{k} \mid x_{1: t}\right)$ for any $k<t$ is calculated recursively using the following equations (Särkkä

227 2013):

$$
\begin{gathered}
p\left(r_{k+1} \mid x_{1: k}\right)=\sum_{r_{k}=0}^{k} p\left(r_{k+1} \mid r_{k}\right) p\left(r_{k} \mid x_{1: k}\right) \\
p\left(r_{k} \mid x_{1: t}\right)=p\left(r_{k} \mid x_{1: k}\right) \sum_{r_{k+1}=0}^{k+1} \frac{p\left(r_{k+1} \mid r_{k}\right) p\left(r_{k+1} \mid x_{1: t}\right)}{p\left(r_{k+1} \mid x_{1: k}\right)} .
\end{gathered}
$$

228 The first equation calculates the one step forward predictions using the change point prior and

229 the posterior probabilities. This equation can be also used sequentially to predict the run length

230 distribution at any time step in the future. The second equation is the backward recursion to 
231 compute the smoothed run length probabilities starting from the latest time step, i.e, $k+1=$ $232 t$.

233

\section{Choosing the prior hyperparameters}

235 Usually in Bayesian context the prior distributions should encapsulate the available expert 236 knowledge of the underlying phenomenon. The presented algorithm is very sensitive to the 237 choice of prior hyperparameters. To make the parametrization of the prior more intuitive we 238 specify the hyperparameters in terms that are more understandable and easier to elicit from 239 experts. We use the upper limit of the variable of interest, $\tilde{u}$, the lower limit of that variable, $\tilde{l}$, 240 the proportion of variance within the regime to the overall variance, $\tilde{\delta}$, and as a measure of 241 uncertainty the coefficient of variation of the regime variance, $\tilde{c}$. By the tilde notation we 242 stress that these quantities represent the expert knowledge and are not derived from data.

243 Since the mean of the next regime is unknown, we use a quite uninformative prior for

244 the mean parameter. This is accomplished by choosing a small $\kappa^{(0)}=0.05$. After choosing a 245 small mean scale parameter the mean location parameter does not have much effect on the 246 posterior distribution of the mean parameter but we set it to be the average of the upper and

247 lower limits, $\gamma^{(0)}=\frac{1}{2}(\tilde{l}+\tilde{u})$. We cannot be as uninformative about the precision parameter, 248 however. We model the expected variance of a regime as a proportion of the overall variance of 249 the data. We do not know in advance what the variance of the data will be, so we make a crude 250 estimate $\tilde{\sigma}_{x}^{2}=\frac{1}{16}(\widetilde{u}-\tilde{l})^{2}$, which corresponds to the range rule of thumb that says that the range is 251 approximately four times the standard deviation. Using the results for the posterior 252 hyperparameters after updating with $n$ data points (see Murphy 2007), the definition of 
253 coefficient of variation, and by approximating the expected variance with our crude estimate,

254 and assuming $\kappa^{(0)} \approx 0$, we can write

$$
\alpha^{(0)}=\frac{1}{\tilde{c}^{2}}+2-\frac{n}{2}
$$

255 and

$$
\beta^{(0)} \approx \frac{1}{16}(\tilde{u}-\tilde{l})^{2} \tilde{\delta}\left(\frac{1}{\tilde{c}^{2}}+1-\frac{n}{2}\right)
$$

256 Next, we arbitrarily choose that the posterior distribution should coincide with the expert

257 opinion when four data points have been used to update the prior knowledge, i.e., set $n=4$ to 258 obtain

$$
\alpha^{(0)}=\frac{1}{\tilde{c}^{2}}
$$

259 and

$$
\beta^{(0)}=\frac{1}{16}(\tilde{u}-\tilde{l})^{2} \tilde{\delta}\left(\frac{1}{\tilde{c}^{2}}-1\right)
$$

260 where it is required that $0<\tilde{c}<\sqrt{\frac{\widetilde{\delta}}{2-\widetilde{\delta}}}$ to ensure that $\beta^{(0)}$ is positive. In our numerical

261 examples, we chose the limits based on the data, and set $\tilde{\delta}=0.25$ and $\tilde{c}=0.1$.

262

263 Detecting regime shifts

264 Preliminary analyses showed that with a suitable choice of hyperparameters the method

265 performs well in producing the posterior predictive distributions and the run length

266 probabilities. However, due to the probabilistic nature of the algorithm, exact timings of the

267 regime shifts are not part of the algorithms output. If used for stock projections, the posterior

268 predictive distribution of BOCPD together with the change point prior hold all relevant

269 information for calculating future predictions, and it is not necessary to know the exact time of 
270 the regime shifts. If the task of detecting the time of the shifts is of interest, we must choose a

271 decision rule based on which the timings of the shifts are to be decided. We choose to give an

272 early indication of a regime shift when the posterior probability of a new run exceeds a

273 threshold of 0.5 , i.e.

$$
p\left(r_{t}=1 \mid x_{1: t}\right)>0.5
$$

274 It is worthwhile to note here that the adopted regime shift indication scheme uses the new data

275 point only to check if there is evidence for a regime shift happening from the previous time step

276 to the current, and it does not update our belief about regime shifts in the past. However, we use

277 the smoothed run length probabilities to check if our belief about regime shifts at time $k$ in the

278 past is updated by the current data point. We thus also indicate a regime shift whenever

$$
p\left(r_{k}=1 \mid x_{1: t}\right)>0.5
$$

and calculate the delay $t-k$. At the end of the time series we also check the most up to date smoothed run length distribution and use it to check for false alarms produced by the posterior or smoothed probabilities during the previous time steps.

\subsection{Model validation with simulated data}

To investigate the model performance, we simulated cod population dynamics under differing overall productivity regimes and then investigated whether the method was able to detect the change points between the regimes correctly. Cod population dynamics were simulated using an individual- and process-based model parameterized for Northern cod (Kuparinen et al. 2012a; Kuparinen and Hutchings 2012). The simulation model tracks a population through time and, at each time step (year), models the processes of natural mortality, growth, maturation, and reproduction for each individual. To incorporate demographic stochasticity to 
291 the population dynamics, the outcomes of the processes are based on random numbers drawn

292 from appropriate distributions. Egg production depended on female body size (May 1967) and

293 was then tuned up or down depending on the population density and environmental

294 year-to-year variability in recruit-per-spawner ratios (number of recruits divided by the

295 spawning stock biomass, R/S). Environmental recruitment variability was modelled through

296 normally distributed random numbers with mean zero and the standard deviation set to the

297 average level of year-to-year variation across 18 cod stocks in the Northern Atlantic

298 (Kuparinen et al. 2014; Keith and Hutchings 2012). Density-dependent recruitment variability

299 was based on the same data, assuming compensatory dynamics. For further detail about the

300 parameterization, see Kuparinen et al. 2014.

301 We simulated cod population dynamics under equilibrium conditions and at annual

302 time steps recorded the recruit-per-spawner ratios. During the course of each 200 year long

303 simulation, we introduced 11 productivity regimes the durations of which varied from 10 to 33

304 years. We further simulated four scenarios for the magnitude of the difference in overall

305 productivity among the regimes, and repeated each scenario 50 times. Altogether we had 200

306 time series with 10 regime shifts in each. This allowed us to explore, how large the differences

307 between the regimes need to be as compared with the demographic stochasticity within the

308 regimes, to detect the shift between two consecutive regimes. We fixed the hazard rate in the

309 change point prior to $\lambda=10$.

310 Because we worked with simulated data we knew the true times of the shifts. Using this

311 knowledge we were able to calculate different metrics to quantify the performance of the

312 method. The known shifts were categorised into three categories based on the indicators

313 produced by the method: 1) shifts that were detected and had their starting year timed correctly,

314 2) shifts that were detected but the starting year of the regime was timed incorrectly, and 3) 
315 shifts that went completely undetected. Sometimes the last data points of a regime were by

316 chance so close to the level of the next regime that the algorithm wrongly located the start of

317 the next regime couple of time steps earlier and, since the following data points were

318 adequately explained by the wrongly timed run, no other indication of the regime shift was

319 produced. Thus, we included in the second category also shifts that were detected one or two

320 time step before they actually happened. For any practical purposes such an error seems

321 acceptable. Since many of the shifts were not detected immediately after the first data point of

322 a new regime was analyzed, we also calculated the delay of the detection, i.e. how many data

323 points from the new regime in addition to the first data point were needed to confirm that a shift

324 had happened. All indications of a shift inside a regime for which the start had already been

325 detected were regarded as false alarms.

326 Since the shifts varied in magnitude, we developed a measure for the "easiness" of

327 detection. We computed the sample mean and sample standard deviation of each regime, and

328 defined the easiness of detection of a shift as the absolute difference between the sample means

329 of two consequtive regimes divided by the sample standard deviation of the previous regime,

$330 E_{i}=\frac{\left|\mu_{i+1}-\mu_{i}\right|}{\sigma_{i}}$. We divided the shifts into five categories based on their measure of easiness: $0-$

$3310.5,0.5-1,1-1.5,1.5-2$, and $>2$.

332

\section{2.3. Model application to empirical data}

334 We further conducted the analysis of the productivity regime shifts using empirical cod

335 productivity time series estimates available at RAM legacy stock assessment database

336 (http://ramlegacy.marinebiodiversity.ca; Ricard et al. 2011). To this end, we investigated

337 recruit-per-spawner estimate time series of four cod stocks that were chosen representative of 
338 stocks that have potentially undergone drastic changes in their productivity. The chosen stocks

339 were Northern cod (COD2J3KL), Southern Gulf of St. Lawrence cod (COD4TVn), North-East

340 Arctic cod (CODNEAR), and North Sea cod (CODNS), and the assessment methods used to

341 produce the recruit and spawning stock biomass estimates were SURBA, VPA/ADAPT,

$342 \mathrm{VPA} / \mathrm{XSA}$, and SAM, respectively. We examined the stock assessment time series visually,

343 and roughly estimated that the expected length of a regime would be 10 years. Thus, we fixed

344 the hazard rate in the change point prior to $\lambda=10$.

\section{3. Results}

\section{3.1. Detection of shifts in simulated data}

348 The method was able to detect shifts in the simulated R/S data. To give an example of the

349 simulated R/S time series, and to illustrate how the posterior predictive distribution obtained

350 during the forward filtering behaves in the model we have plotted the first 50 data points of a

351 randomly chosen simulation time series (Fig. 1). It is easy to notice how the posterior

352 predictive quickly reacts to the regime shifts, whereas the sample mean changes very slowly

353 and is not very representative of the regimes after the shifts (Fig. 1a). To clarify the concept of

354 the run length, we have also plotted the smoothed run length probabilities (Fig. 1b).

355 A summary of the method's performance is given in Table 1. The method produced 639

356 false alarms (33.1\% of all indications). To illustrate how the easiness of the shift affects the

357 method's ability to detect the shifts we have plotted the detection frequencies for each easiness

358 category (Fig 2a). As expected, the detection frequency increases as the easiness of the shifts

359 increases. We also plotted the detection delay as a function of the easiness measure (Fig $2 b$ ).

360 The highest detection delays occur for the more difficult shifts. The median delay in the 
361 simulations was one, and $76 \%$ of the delays were at most three. There are not many points on

362 the far left since only few shifts having very low easiness measure were detected.

363

364

\subsection{Detection of shifts in empirical data}

365 The method was able to detect shifts in the empirical data. The posterior predictive

366 distributions obtained during the forward filtering, and the probabilities for regime shifts are

367 presented in Figure 1. For the Northern Cod the method found three shifts. The first shift was

368 found in year 2000 with a three year delay. The second shift was found in real time in 2002 and

369 the third in real time in 2006. After the whole time series was analyzed the final smoothing

370 showed that only the shifts in 2002 and 2006 had higher probability than the set threshold of 0.5

371 (Fig 3a). To investigate how easy the shifts were in terms of the easiness measure, we

372 calculated the easiness measure for each detected shift. The easiness for the shifts in Northern

373 Cod time series were $E_{1}=3.2$ and $E_{2}=4.1$.

374 For the Southern Gulf of St. Lawrence cod, six shifts were found (Fig. 3b). The first

375 shift in 1972 was found with a one year delay, the next shifts in 1973 and 1980 were found in

376 real time. The next shift was found in 1992 with a two year delay, and the next shift in 1993 was

377 found in real time. The last shift in 1996 was found with a six year delay. After the final

378 smoothing only the shifts in 1973, 1980, 1993 and 1996 had higher probability than the set

379 threshold, and the corresponding values of the easiness measure were $E_{1}=2.8, E_{2}=4.8$,

$380 E_{3}=3.9$ and $E_{4}=3.8$, respectively.

381 Only two shifts were found for the North-east Arctic cod (CODNEAR). The shifts

382 happened in 1965 and 1968 and were found in real time, and were still regarded as shifts after 
383

384

385

386

387

388

389

390

391

392

393

394

395

396

397

398

399

400

401

402

403

404

405

the final smoothing (Fig. 3c). The easiness measures for the detected shifts were $E_{1}=8.2$ and $E_{2}=3.2$, respectively.

Fourteen shifts were found for the North Sea cod (CODNS) (Fig. 3d). The first two shifts in 1967 and 1969 were found with a one year delay, and the third shift in 1971 was found with a two year delay. The next six shifts in 1976, 1980, 1985, 1986, 1991 and 1993 were found in real time. The next shift in 1994 was found with a one year delay, and the last four shifts in 1996, 1997, 1999 and 2005 were found in real time. After the final smoothing only eight of the shifts $(1976,1980,1985,1986,1991,1994,1996$ and 1997) had higher probability than the threshold level of 0.5 . In this case the parameterization of our method clearly failed since so many shifts were found so close to each other. Since our measure of easiness cannot be calculated for regimes of length one, we could not report those for the North Sea cod.

The easiness measures for the shifts found in the empirical data ranged from 2.8 to 8.2. Visual inspection of the time series does not suggest that any other shifts exist. So if this level of easiness is to be expected, the method will most likely find all the shifts. In light of this, most of the shifts in the simulated time series were more difficult to identify than in the empirical data.

\section{Discussion}

In the present study, we have used Bayesian online change point detection algorithm (Adams and MacKay 2007) to detect regime shifts in fish stock productivity. Most of the previous attempts to detect regime shifts in stock productivity have been based on frequentist methods (e.g., Vert-Pre et al. 2013; Wayte 2013). It is not clear, however, how these methods account 
406 for the uncertainty in their estimated locations of the regime shifts or the uncertainty in the

407 estimated parameters of each regime. Furthermore, it is not clear how these uncertainties would

408 be accounted for in projections of the future state of the stock. In the Bayesian method

409 presented in this study, the uncertainty is present in all of the parameter estimates, as well as in

410 the locations of the shifts, and the uncertainties are represented by probability distributions that

411 reflect the modeler's degree of belief. Projections of the stock would be carried out using the

412 posterior predictive distribution, which incorporates all the aforementioned uncertainties. The

413 uncertainties depend on the choice of the prior hyperparameters, the hazard rate, and the

414 underlying predictive model, but all these assumptions are presented in a transparent manner

415 and are subject to scrutiny. The difficulty with Bayesian methods arises when choosing the

416 prior hyperparameters, since in many occasions the hyperparameters can be very difficult to

417 interpret and to tune to the study in question. However, methods to estimate the

418 hyperparameters from training data have also been developed (Turner et al. 2009).

420 4.1. Connections with previous work on cod productivity

421 The Northern cod has been shown to have collapsed solely because of overfishing in 1992

422 (Hutchings and Myers 1994), but migration due to changes in sea water temperatures and

423 decline of capelin has been demonstrated to have co-occurred with the same event (Rose et al.

424 2000). Our dataset starts at 1991 and cannot answer any questions about the pre-collapse state

425 of the stock. However, it seems that there was a shift in R/S to a higher level in 2002, but

426 another shift has occurred in 2006 to very low levels of R/S.

427 The late 1980s regime shift in the Gulf of St. Lawrence ecosystem has been attributed

428 to human exploitation of cod and redfish which resulted in a major perturbation of the structure 
429 of the ecosystem from one previously dominated by demersal and small-bodied forage species

430 to one now dominated by small-bodied forage species (Savenkoff et al. 2007). Previous

431 analyses on productivity regimes in the Gulf of St. Lawrence cod have identified three

432 productivity regimes spanning the years 1950-1972, 1973-1987, and 1988-2002 (Mohn and

433 Chouinard 2007). Changes in the productivity of Gulf of St. Lawrence cod over the past few

434 decades have been attributed to changes in natural mortality and growth (Chouinard et al.

435 2006). The production has been suggested to be linked to changes in the ambient temperature

436 through declining size-at-age (Mohn and Chouinard 2004; Dutil et al. 1999). Also,

437 size-selective fishing has been suggested to have caused genetic changes in growth in this

438 population (Swain et al. 2007). Our analysis proposes that there was a shift to higher R/S

439 regime in 1973, which is consistent with the findings of Mohn and Chouinard (2007). The

440 second shift to a lower regime was found by our method in 1980, which is several years earlier

441 than the low productivity regime proposed by Mohn and Chouinard (2007). Our method

442 proposes that a shift to higher R/S regime happened also in 1993 but shift back to lower regime

443 happened in 1996. It may be, however, questionable to define such a short period as a regime.

444 According to Brunel and Boucher (2007) the lower recruitment of North-east Arctic

445 cod after the late 1980 s could be attributed to elevated water temperatures. The recruitment of

446 North-east Arctic cod has also been subject to earlier study (Godø 2003), which concluded that

447 strong year-classes were produced in 1948-1950, 1963-1964, 1969-1970, and 1983. Godø

448 (2003) suggests that the stock is affected by long-term fluctuations in the environment. Our

449 method found shifts in R/S to a higher level in 1965 and back to a lower level in 1968. The

450 findings correspond to the strong year-classes of 1696-1970 found by Godø (2003).

451 Positive correlation between the low recruitment of gadoids in the North Sea and strong

452 positive northern hemisphere temperature anomalies after 1987 has been established 
453 (Beaugrand 2004, O'Brien et al. 2000). The fluctuations in the abundance of plankton have

454 been stated as one of the causes of long-term changes in cod recruitment (Beaugrand et al.

455 2003). Our analysis suggests that the North Sea cod stock entered a higher R/S regime in 1976,

456 shifted back to a lower R/S regime in 1980, and has been fluctuating greatly during the period

457 1991 - 1996, and has been in a low R/S regime since 1997. However, our method essentially

458 failed for the North Sea cod, mainly because the proportion of the within regime variance to the

459 overall variance was underestimated while parameterizing the model.

460

\section{$461 \quad 4.2$. On indicators of a regime shift}

462 Charasteristic to the Bayesian algorithms is that their output is a propability distribution as

463 opposed to the point estimates produced by deterministic or frequentist methods. However, 464 sometimes some kind of an indicator of the regime shifts is desirable. Adams and McKay 465 (2007) did not discuss the problem of explicitly inferring the locations of the change points in 466 the data. The algorithm solves the run length posterior probability distribution and the posterior 467 probability distributions of the parameters for each run length, and if an indicator of a regime 468 shifts is needed, it is up to the user to choose a utility function and find the decision ("regime 469 shift occurred" or "no regime shift") that maximizes the expected utility. However, the

470 development of a suitable utility function from management's perspective is out of the scope of

471 this study. For simplicity, we use the posterior run length probabilities to give an early

472 indication of a regime shift based on the newest data point and use the smoothed run length

473 probabilities for retrospective regime shift indication. We indicate a regime shift when the

474 probability of a run length of one is greater than a threshold value of 0.5 . That is, the probability 
475 that the data point of the particular time step is the first in a new regime is greater than the

476 probability of the data point belonging to any of the possible previous regimes.

477 The success of early regime shift detection based on the posterior run length

478 probabilities depends heavily on the prior hyperparameters, the hazard rate, and the threshold

479 value. If the hyperparameters controlling the precision of a new regime are set so that the

480 regime specific posterior predictive distributions become very narrow, even small deviations

481 from the mean get interpreted as regime-shifts, and on the other extreme if the predictive

482 distributions have very high uncertainty, even large deviations are accepted as belonging to the

483 previous regime. Using the smoothed run length probabilities improves the accuracy of regime

484 shift detection because it uses more data to evaluate the probabilities. Smoothing, however, can

485 be only used to improve estimates of regime shifts in the past, and this introduces delay to the

486 regime shift detection. In our simulations the median delay was one, and $76 \%$ of the delays

487 were at most three.

488

489 4.3. Model limitations

490 In our case, the data generating process was Gaussian with unknown mean and variance. We

491 recognize, however, that the Gaussian distribution cannot accurately model

492 recruit-per-spawner data since it always has some probability mass on the negative real axis,

493 and it is impossible for a stock to produce a negative number of recruits. We considered

494 working with the log-normal distribution but the occasional years of zero recruitment in our

495 simulation model made this impossible. Thus, we used Gaussian distribution and feel that it

496 serves well for the purpose of demonstrating the method. The BOCPD algorithm works with

497 any data generating process that is able to provide posterior predictive probabilities. If the 
498 likelihood is in the exponential family and a conjugate prior is used, the posterior can be solved 499 analytically and is computed by recursively updating the sufficient statistics since the last 500 potential change point. This recursive approach has very low computation and memory

501 requirements. Thus, other distributions belonging to the exponential family should be explored 502 in the future to find the most realistic likelihood for the variable under investigation.

503 Distributions not belonging to the exponential family might be also considered but they tend to

504 lead to problems where the exact solution requires computationally expensive sampling

505 methods, such as Markov Chain Monte Carlo. This is undesirable since many complex

506 Bayesian stock assessment models already suffer from computational problems (Hilborn 2003;

507 Kuparinen et al. 2012b), and integrating change point models into stock assessment models

508 will further increase the amount of computation needed. However, variational approximations

509 to non-exponential family change point models have been developed recently (Turner et al.

510 2013), and could also be explored.

511 We have used recruit-per-spawner ratios from existing model outputs to identify

512 productivity regime shifts. This approach is very limited, since looking only at R/S does not

513 take into account compensatory or depensatory effects. However, the widely applied Ricker

514 stock-recruitment model can be formulated as a linear regression model that could be used as

515 the data generating process with BOCPD to increase biological realism without sacrificing the

516 analytical tractability of the algorithm. Moreover, productivity also depends on the mortality

517 and growth of the population. We did not include them in our study, however, and believe that

518 using this simple approach the operation of the methods is easier to present in an

519 understandable format. However, as mentioned earlier, BOCPD works with any UPM, and in

520 cases where the data model can be described as a linear regression, conjugate priors can be used

521 to preserve the analytical tractability and computational efficiency of the algorithm. It is quite 
522 straight-forward to extend the method for multi-dimensional data also to simultaneously

523 analyze several variables for the same stock or to analyze several stocks at once to find

524 temporal patterns across stocks. Moreover, our model does not include autocorrelation

525 between consequtive R/S estimates, but instead assumes that they are mutually idependent, and

526 the parameters of the data generating process are constant within each regime.

527 We also acknowledge that the models that have produced the recruit-per-spawner

528 estimates used in this study include their own assumptions about the dynamics of the

529 population that can affect the estimates in form of biases and correlation between the estimates.

530 For a detailed discussion about the pitfalls of post-hoc analyses of stock assessment model

531 outputs see (Brooks 2015). Thus, given the aforementioned limitations of our approach, we

532 propose not to take the results for the four cod stocks presented in this study as definitive proof

533 of shifts in the productivity of these stocks, but rather as numerical illustrations of the BOCPD

534 methodology which has not been used in fisheries reseach before.

535 In some ecosystems, it might be reasonable to model the system so that it switches

536 between two or more reoccuring regimes, although we believe that the assumption that exactly

537 the same conditions would repeat in reality are somewhat questionable considering the huge

538 amount of different factors that affect the ecosystem. However, periodic recruitment has been

539 observed for example in the Pacific mackerel fishery, and the periodicity has been contributed

540 to periodically changing sea level, barometric pressure and sea surface temperature (Parrish

541 and MacCall 1978). Our model is not designed for these kinds of situations since it is assumed

542 that the parameters of the new regime depend only on the recent data and not on the data

543 belonging to any previous regimes. Thus Markov switching regression models (Munch and

544 Kottas 2009) will likely outperform BOCPD in cases where the system switches between

545 reoccurring regimes. It is, however, possible to include our knowledge about the previous 
546 regimes in the prior distribution for a new regime in our model. A straight-forward approach

547 would be to use a mixture of previously identified regimes as the prior for new regime. The

548 weights for each regime could indicate the relative frequencies of the different regimes, and as

549 the hyperparameters we could use the posterior hyperparameters obtained for each regime

550 earlier. This kind of mixture distribution approach would increase computation exponentially

551 since at each time step each mixture component would essentially produce its own single

552 component 'path' with equivalent complexity of the presented single component prior model.

553 However, an approximate version where the components with very low probability would be

554 'forgotten' could be used to restrict the computational demand to acceptable levels. This

555 approach has not been investigated yet but seems conceptually solid, and would probably

556 improve regime shift detection considerably in systems where the regimes reoccur.

557

\section{4.4. Implications for stock assessment}

559 In this study, we focused on detecting productivity regime shifts in historical stock assessment

560 data using estimates of the number of recruits and spawning stock biomass. Our approach was

561 to inspect the posterior and smoothed run length probabilities for signs of regime shifts. This

562 kind of an approach can provide valuable information about the magnitude and frequency of

563 the productivity regimes experienced by a stock in the past, but it can also be used as a time

564 series analysis tool to warn about coming regime shifts whenever new assessment data are

565 available. However, we believe that the real potential of the method lies in incorporating the

566 change point model of BOCPD directly into Bayesian stock assessment models. Any model

567 parameter that is traditionally modeled as a stationary process but shows regime-type

568 behaviour could be modeled as a change point process using the approach studied here. 
569 Especially if there is considerable variation between regimes, the posterior predictive

570 distribution of BOCPD model differs greatly from the posterior predictive distribution of a

571 model that assumes no regime shifts, and is more up-to-date regarding the recent development

572 in the stock status. Traditionally in stock assessment models, the density-dependent

573 productivity is assumed constant across years, and the stock-recruitment model parameters are

574 estimated using all available historical data (Hilborn 2003; Kuparinen et al. 2012b). Using

575 change point models, the assessments may take into account the fact that under different

576 productivity regimes the model parameters can change very rapidly and, thus, either restrict the

577 use of the data to the current regime or simply use the posterior distribution of BOCPD, which

578 implicitly accounts for the regime shifts. This should lead to better estimates of the model

579 parameters because data from past regimes would not affect the estimates related to the current

580 regime as much as when assuming stationary parameters. Implementation of such model

581 changes is still an open problem and warrants for further research.

582

583 4.5. Implications for stock projections

584 In the recent assessments of all of the stocks chosen for this study the future projections are

585 based on the recent estimates of the number of recruits or recruit-per-spawner ratios. In the

5862012 assessment of cod in the Southern Gulf of St. Lawrence, the projections are based on an

587 assumption of a linear stock-recruit relationship, and the slope of the relationship is chosen

588 randomly from the R/S estimates for years 1994-2009 (Swain et al. 2012). The 2013

589 assessment of Northern Cod calculates projections to 2016 assuming that the future

590 recruitment is the average of the past three years (DFO 2013). In the 2012 assessment of the

591 North-east Arctic Cod, the projection for 2015 was carried out using the long-term arithmetic 
592 mean of estimated recruitment (ICES 2012a). Whereas, in the 2011 assessment of the North 593 Sea Cod, the future recruitment is re-sampled from the recruitment estimates of 1997 to the 594 most recent year classes (ICES 2012b). All of these projections assume very little or no 595 uncertainty at all in the predicted future recruitment, and implicitly assume perfect knowledge 596 about the current regime and about its continuation in the near future. We believe that the 597 methods proposed in this study could be immediately applied for stock projections by using the 598 posterior predictive distribution of the latest year to predict the recruitment of the next year, 599 and then by the sequential application of the one step forward predictions (13) calculate the 600 predicted recruitment for the subsequent years. This would include a more realistic measure of 601 uncertainty for developing future stock projections.

602

\section{Acknowledgements}

604 This study was funded by the Academy of Finland (through a grant to AK). We thank Samu

605 Mäntyniemi, Jarno Vanhatalo, three anonymous referees and the associate editor for helpful 606 comments on an ealier version of the manuscript.

607 
608

609

610

611

612

613

614

615

616

617

618

619

620

621

622

623

624

625

626

627

628

629

\section{References}

Adams, R. P., and MacKay, D. J. C. 2007. Bayesian online changepoint detection. Technical report, University of Cambridge, Cambridge, UK.

Alheit, J., Möllmann, C., Dutz, J., Kornilovs, G., Loewe, P., Mohrholz, V., and Wasmund, N. 2005. Synchronous ecological regime shifts in the central Baltic and the North Sea in the late 1980s. ICES Journal of Marine Science: Journal du Conseil 62(7): $1205-1215$.

Beaugrand, G., Brander, K. M., Lindley, J. A., Souissi, S., and Reid, P. C. 2003. Plankton effect on cod recruitment in the North Sea. Nature 426(6967): 661-664.

Beaugrand, G. 2004. The North Sea regime shift: evidence, causes, mechanisms and consequences. Progress in Oceanography 60(2): 245-262.

Biggs, R., Carpenter S. R., and Brock, W. A. 2009. Turning back from the brink: detecting an impending regime shift in time to avert it. Proceedings of the National academy of Sciences 106(3): 826-831.

Brooks, E. N., and Deroba, J. J. 2015. When "data" are not data: the pitfalls of post-hoc analyses that use stock assessment model output. Canadian Journal of Fisheries and Aquatic Sciences, (ja).

Brunel, T., and Boucher, J. 2007. Long-term trends in fish recruitment in the north-east Atlantic related to climate change. Fisheries Oceanography 16(4): 336-349.

Chouinard, G. A., Currie, L., Poirier, G. A., Hurlbut, T., Daigle, D., and Savoie, L. 2006. Assessment of the southern Gulf of St Lawrence cod stock. Research document 006/006, Canadian Science Advisory Secretariat. 
630 DeYoung, B., Harris, R., Alheit, J., Beaugrand, G., Mantua, N., and Shannon, L. 2004.

631 Detecting regime shifts in the ocean: data considerations. Progress in Oceanography

632 60(2): $143-164$.

633

DFO. 2013. Stock Assessment of Northern (2J3KL) Cod in 2013. DFO Can. Sci. Advis. Sec.

634 Sci. Advis. Rep. 2013/014.

Ditlevsen, P. D. 2012. Robust seasonal cycle of Arctic sea ice area through tipping point in amplitude. arXiv preprint arXiv:1209.3613.

Dutil, J. D., Castonguay, M., Gilbert, D., and Gascon, D. 1999. Growth, condition, and environmental relationships in Atlantic cod (Gadus morhua) in the northern Gulf of St. Lawrence and implications for management strategies in the Northwest Atlantic. Canadian Journal of Fisheries and Aquatic Sciences 56(10): 1818—1831.

Georgescu, V. 2012. Online change-point detection in financial time series: challenges and

Godø, O. R. 2003. Fluctuation in stock properties of north-east Arctic cod related to long-term environmental changes. Fish and Fisheries 4: 121-137.

647 Hilborn, R. 2003. The state of the art in stock assessment: Where we are and where we are 648 going. Scientia Marina 67(S1): 15-20.

649 Holbrook, S. J., Schmitt, R. J., and Stephens Jr, J. S. 1997. Changes in an assemblage of 650 temperate reef fishes associated with a climate shift. Ecological Applications 7(4):

651 $1299-1310$. 
652 Hutchings, J.A. and Myers, R.A. 1994. What can be learned from the collapse of a renewable 653 resource - Atlantic cod, Gadus morhua, of Newfoundland and Labrador. Canadian 654 Journal of Fisheries and Aquatic Sciences 51: 2126-2146.

655 ICES. 2012a. Report of the Arctic Fisheries Working Group 2012 (AFWG), 20 - 26 April 656 2012, ICES Headquarters, Copenhagen. ICES CM 2012/ACOM:05. 670 pp.

657 ICES. 2012b. Report of the Working Group on the Assessment of Demersal Stocks in the 658 North Sea and Skagerrak (WGNSSK), 4 - 10 May 2011, ICES Headquarters, 659 Copenhagen. ICES CM 2011/ACOM:13. 1197 pp.

660 Keith, D. M., and Hutchings, J. A. 2012. Population dynamics of marine fishes at low 661 abundance. Canadian Journal of Fisheries and Aquatic Sciences, 69(7): 1150-1163.

662 Kuparinen, A., Hardie, D. C., and Hutchings, J. A. 2012a. Evolutionary and ecological 663 feedbacks of the survival cost of reproduction. Evolutionary Applications 5: 245-255. 664 Kuparinen, A., and Hutchings, J. A. 2012. Consequences of fisheries-induced evolution for 665 population productivity and recovery potential. Proceedings of the Royal Society B 666 279: $2571-2579$.

667 Kuparinen, A., Mäntyniemi, S., Hutchings, J. A., and Kuikka, S. 2012b. Increasing biological 668 realism of fisheries stock assessment: towards hierarchical Bayesian methods. $669 \quad$ Environmental Reviews 20(2): 135-151.

670 Kuparinen, A., Keith, D. M., and Hutchings, J. A. 2014. Increased environmentally driven 671 recruitment variability decreases resilience to fishing and increases uncertainty of 672 recovery. ICES Journal of Marine Science: Journal du Conseil, fsu021.

673 Liu, P. 2010. Maximum likelihood estimation of an unknown change-point in the parameters 674 of a multivariate Gaussian series with applications to environmental monitoring. Ph.D. 675 thesis, Washington State University. 
676 Malladi, R., Kalamangalam, G. P., and Aazhang, B. 2013. Online Bayesian change point 677 detection algorithms for segmentation of epileptic activity. In Proceedings of the 2013

678 Asilomar Conference on Signals, Systems and Computers, IEEE, pp. 1833-1837.

679 May, A. W. 1967. Fecundity of Atlantic cod. Journal of the Fisheries Board of Canada, 24(7): $680 \quad 1531-1551$.

681 Mohn, R. K., and Chouinard, G. A. 2004. Production analysis of southern Gulf of St. Lawrence 682 cod for the identification of biological reference points. Research Document 2004/039, 683 Canadian Science Advisory Secretariat

684 Mohn, R. K., and Chouinard, G. A. 2007. Harvest control rules for stocks displaying dynamic 685 production regimes. ICES Journal of Marine Science 64: 693 - 697.

686 Munch, S. B., and Kottas, A. 2009. A Bayesian modeling approach for determining 687 productivity regimes and their characteristics. Ecological Applications 19(2): $688 \quad 527-537$.

689 Murphy, K. P. 2007. Conjugate Bayesian analysis of the Gaussian distribution. Technical 690 report, University of British Columbia.

691 Parrish, R. H., and MacCall, A. D. 1978. Climatic variation and exploitation in the Pacific 692 mackerel fishery. Calif. Dept. Fish and Game Fish Bull. 167: 1-110

693 O'Brien, C. M., Fox, C. J., Planque, B., and Casey, J. 2000. Fisheries: climate variability and $694 \quad$ North Sea cod. Nature 404(6774): 142-142.

695 Peterson, W. T., and Schwing, F. B. 2003. A new climate regime in northeast Pacific 696 ecosystems. Geophysical Research Letters 30(17): 1896-1899.

697 Rodionov, S. N. 2004. A sequential algorithm for testing climate regime shifts. Geophysical $698 \quad$ Research Letters 31(9). 
699 Rose, G.A., deYoung, B., Kulka, D.W., Goddard, S.V. and Fletcher, G.L. 2000. Distribution

700 shifts and overfishing the northern cod (Gadus morhua): a view from the ocean.

$701 \quad$ Canadian Journal of Fisheries and Aquatic Sciences 57: 644-663.

702 Ricard, D., Minto, C., Jensen, O. P., and Baum, J. K. 2011. Examining the knowledge base and 703 status of commercially exploited marine species with the RAM Legacy Stock

704 Assessment Database. Fish and Fisheries 13(4): 380-398.

705 Savenkoff, C., Castonguay, M., Chabot, D., Hammill, M. O., Bourdages, H., and Morissette, L.

706 2007. Changes in the northern Gulf of St. Lawrence ecosystem estimated by inverse

707 modelling: evidence of a fishery-induced regime shift?. Estuarine, Coastal and Shelf

$708 \quad$ Science 73(3): 711-724.

709 Särkkä, S. 2013. Bayesian filtering and smoothing. Cambridge University Press.

710 Swain, D. P., Sinclair, A. F., and Hanson, J. M. 2007. Evolutionary response to size-selective

711 mortality in an exploited fish population. Proceedings of the Royal Society B:

$712 \quad$ Biological Sciences 274(1613): 1015-1022.

713 Swain, D. P., Savoie, L., and Aubry, É. 2012. Recovery Potential Assessment for the

714 Laurentian South designatable unit of Atlantic Cod (Gadus morhua): the southern Gulf

715 of St. Lawrence cod stock (NAFO Div. 4T-4Vn(Nov-Apr)). DFO Can. Sci. Advis. Sec.

$716 \quad$ Res. Doc. 2012/052. iii +51 p.

717 Turner, R., Saatci, Y., and Rasmussenm C. E. 2009. Adaptive sequential Bayesian change

718 point detection. In Proceedings of the Temporal Segmentation Workshop at NIPS

719 2009, Whistler, BC, Canada, December 2009, pp. 1-4.

720 Turner, R. 2010. Bayesian change point detection for satellite fault prediction. In proceedings

721 of Interdisciplinary Graduate Conference (IGC) 2010, Cambridge, UK, June 2010, pp.

$722 \quad 213-221$. 
723 Turner, R. D., Bottone, S., and Stanek, C. J. 2013. Online variational approximations to

724 non-exponential family change point models: with application to radar tracking. In

725 proceedings of the Advances in Neural Information Processing Systems 26 (NIPS

726 2013), Lake Tahoe, Nevada, December 2013, pp. 306-314.

727 Vert-pre, K. A., Amoroso, R. O., Jensen, O. P., and Hilborn, R. 2013. Frequency and intensity

728 of productivity regime shifts in marine fish stocks. Proceedings of the National

729 Academy of Sciences 110(5): 1779—1784.

730 Wang, N. C., Hudson, R. E., Tan, L. N., Taylor, C. E., Alwan, A., and Yao, R. 2013. Change

731 point detection methodology used for segmenting bird songs. In Proceedings of the

2013 IEEE China Summit \& International Conference on Signal and Information

733 Processing, Beijing, China, July 2013, pp. 206-209.

734 Wayte, S. E. 2013. Management implications of including a climate-induced recruitment shift

735 in the stock assessment for jackass morwong (Nemadactylus macropterus) in

736 south-eastern Australia. Fisheries Research 142: 47-55. 
737 Table 1. Summary of the regime shift detection in simulated Atlantic cod recruit-per-spawner

738 time series. The reported quantities in each easiness class are the proportion of shifts detected

739 at the correct time, the median detection delay for the correctly detected shifts, the proportion

740 of shifts that were detected at a wrong time, the median error in time for the wrongly timed

741 shifts, and the proportion of shifts that went undetected.

\begin{tabular}{c|c|c|c|c|c} 
Easiness class & $\begin{array}{c}\text { Correct } \\
\text { time (\%) }\end{array}$ & $\begin{array}{c}\text { Median } \\
\text { Delay }\end{array}$ & $\begin{array}{c}\text { Wrong } \\
\text { time (\%) }\end{array}$ & $\begin{array}{c}\text { Median } \\
\text { Error }\end{array}$ & $\begin{array}{c}\text { Missed } \\
(\%)\end{array}$ \\
\hline \hline $0-0.5$ & 1.3 & 1 & 20.4 & 5 & 78.3 \\
\hline $0.5-1.0$ & 11.1 & 3 & 26.7 & 3 & 62.2 \\
\hline $1.0-1.5$ & 25.3 & 2 & 25.0 & 2 & 49.7 \\
\hline $1.5-2.0$ & 41.5 & 2 & 33.1 & 2 & 25.4 \\
\hline$>2.0$ & 81.0 & 1 & 12.1 & 1 & 6.9
\end{tabular}

742 
743 Figure legends
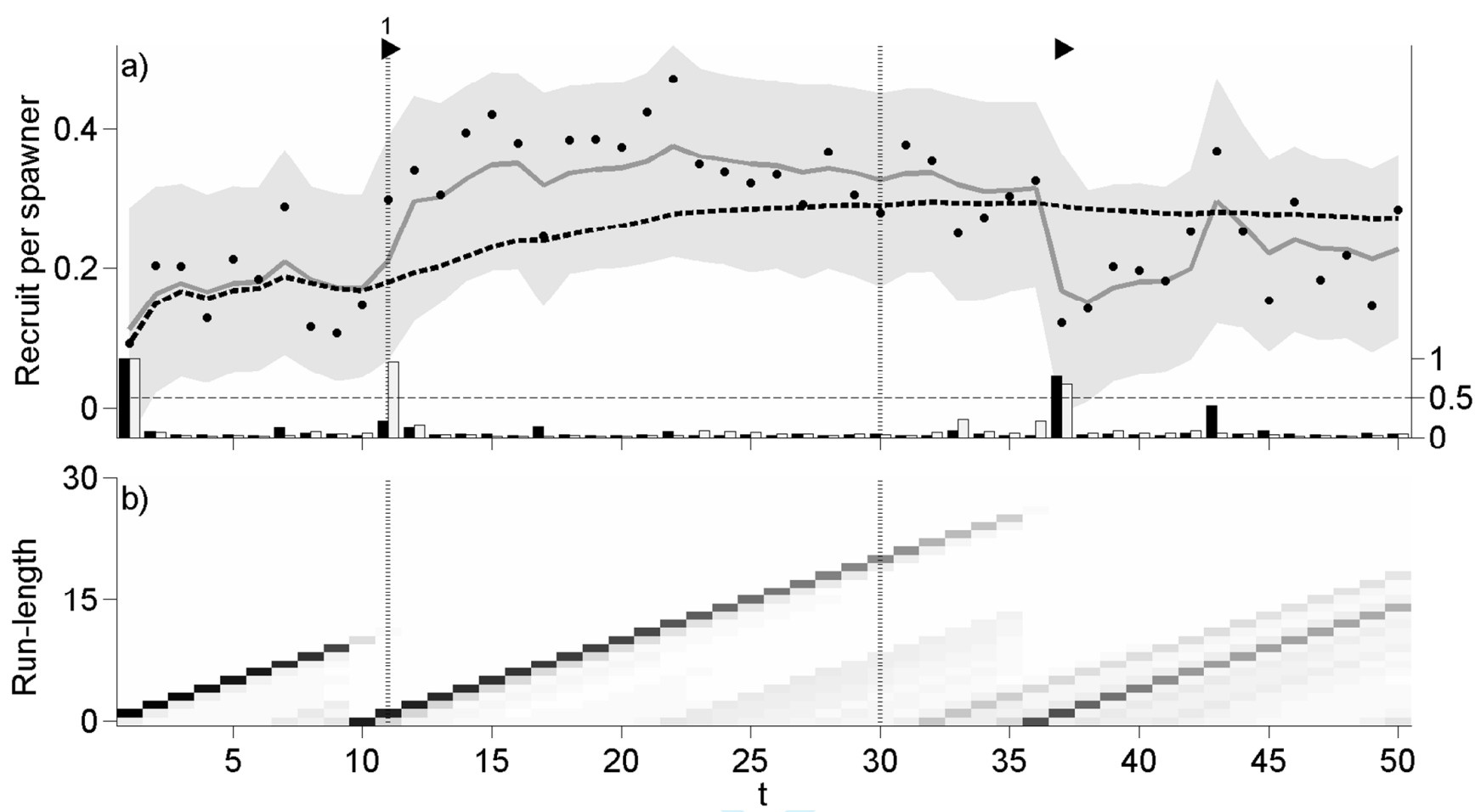

744 Figure 1. Illustration of the method performance using the simulated recruit-per-spawner time

745 series. On the top row (panel a) we have plotted the recruit-per-spawner data (black dots), the

746 mean of the posterior predictive distribution of the current time step obtained during the

747 forward filtering (gray solid line), the $90 \%$ probability interval of the posterior predictive

748 distribution (shaded gray area), the sample mean calculated by using all the data up to the

749 current time step (dashed black line), the years where a start of a new regime were found based

750 on the posterior run length probabilities (black triangles) or smoothed run length probabilities

751 (black triangles with a number on top indicating the detection delay), the years of actual regime

752 shifts (dashed vertical lines), the posterior probabilities for a start of a new regime (black bars),

753 the final smoothed probabilities for a start of a new regime after using all the data (light gray

754 bars), and the threshold probability level of 0.5 for the regime shifts (dashed horizontal line).

755 The second row (panel b) shows the final smoothed run length distribution. Darker color 
756 indicates higher probability. The plot is cropped so that only run lengths up to 30 are shown.

757 Run lengths greater than 30 had negligible probabilities.

758

a)

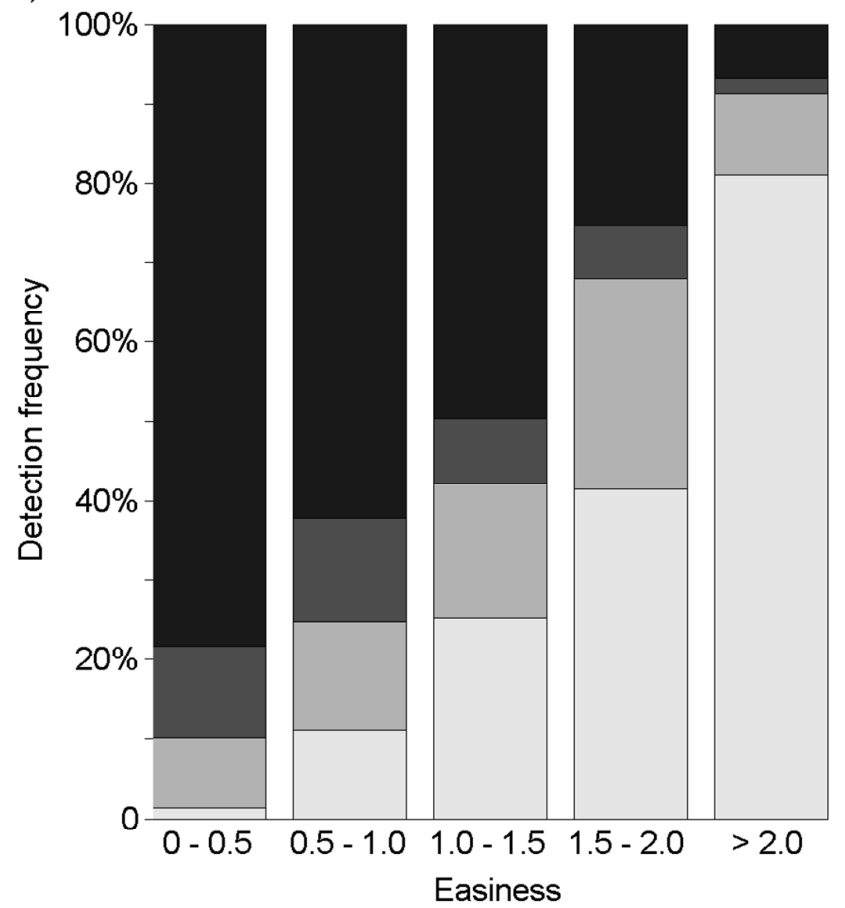

b)

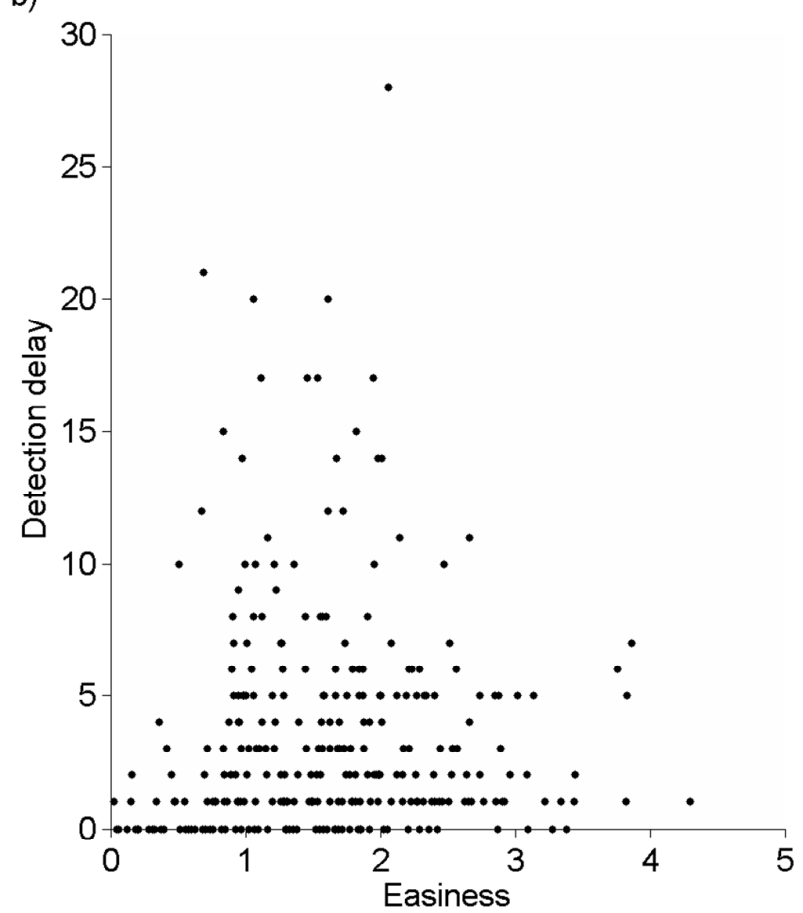

759 Figure 2. Examination of the performance of the method as a function of the easiness measure

760 for the simulated recruit-per-spawner data (number of recruits divided by spawning stock

761 biomass). The shifts are divided in four categories: the shifts that were detected correctly

762 (lightest gray), the detected shifts for which the error in time was at most three time steps

763 (medium gray), the detected shifts for which the error in time was greater than three time steps

764 (darkest gray), and the shifts that were missed completely (black). The frequencies of each

765 category are shown in panel a. The frequency data is grouped into five levels of easiness. Panel

$766 \mathrm{~b}$ shows the detection delay versus the easiness measure for the shifts that were detected

767 correctly or with an error in time of at most three time steps.

768 


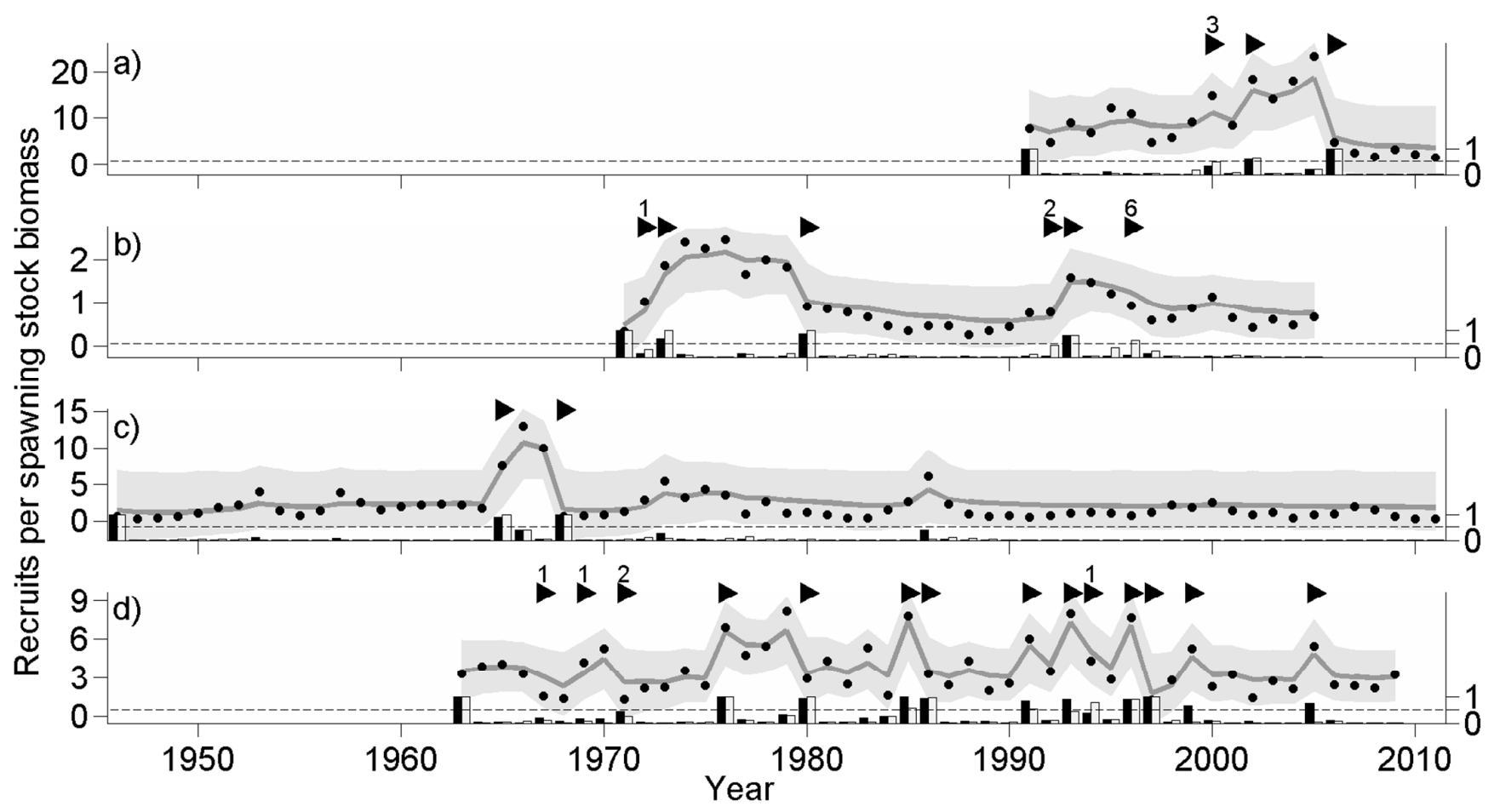

769 Figure 3. Illustration of the method using the recruit-per-spawner estimates for four Atlantic

770 cod stocks. The stocks used in the analysis are a) Northern Cod (COD2J3KL), b) Southern Gulf

771 of St. Lawrence (COD4TVn), c) North-east Arctic (CODNEAR) and d) North Sea (CODNS).

772 The year is shown on the horizontal axis and the recruit-per-spawner ratio (number of recruits

773 divided by spawning stock biomass) on the vertical axis. We have plotted the

774 recruit-per-spawner estimates (black dots), the mean of the posterior predictive distribution of

775 the current time step obtained during the forward filtering (solid gray line), the $90 \%$

776 probability interval of the posterior predictive distribution (shaded gray area), the years where

777 a start of a new regime were found based on the posterior run length probabilities (black

778 triangles) or smoothed run length probabilities (black triangles with a number on top indicating

779 the delay), the posterior probabilities for a start of a new regime (dark gray bars), the final 
780 smoothed probabilities for a start of a new regime after using all the data (light gray bars), and 781 the threshold probability level of 0.5 for the regime shifts (dashed horizontal line). 


\section{Appendix A}

2 The recursive computation of the joint probability distribution of the run length and the 3 data

$$
\begin{aligned}
p\left(r_{t}, x_{1: t}\right)= & \sum_{r_{t-1}=0}^{t-1} p\left(r_{t}, r_{t-1}, x_{t}, x_{1: t-1}\right)=\sum_{r_{t-1}=0}^{t-1} p\left(r_{t}, x_{t} \mid r_{t-1}, x_{1: t-1}\right) p\left(r_{t-1}, x_{1: t-1}\right) \\
& =\sum_{r_{t-1}=0}^{t-1} p\left(r_{t} \mid r_{t-1}, x_{1: t-1}\right) p\left(x_{t} \mid r_{t-1}, x_{1: t-1}\right) p\left(r_{t-1}, x_{1: t-1}\right) \\
& =\sum_{r_{t-1}=0}^{t-1} p\left(r_{t} \mid r_{t-1}\right) p\left(x_{t} \mid x_{\left(t-r_{t-1}\right):(t-1)}\right) p\left(r_{t-1}, x_{1: t-1}\right) \\
& =\sum_{r_{t-1}=0}^{t-1} p\left(r_{t} \mid r_{t-1}\right) p\left(x_{t} \mid x^{\left(r_{t-1}\right)}\right) p\left(r_{t-1}, x_{1: t-1}\right)
\end{aligned}
$$

4 The probability density function of the non-standardized Student's t-distribution

$$
t_{v}(x \mid \xi, \omega)=\frac{\Gamma\left(\frac{v+1}{2}\right)}{\Gamma\left(\frac{v}{2}\right) \sqrt{\pi v \omega}}\left(1+\frac{(x-\xi)^{2}}{v \omega}\right)^{-\frac{v+1}{2}}
$$

5 The posterior predictive probability distribution

$$
\begin{aligned}
p\left(x_{t+1} \mid x_{1: t}\right)= & \sum_{r_{t}=0}^{t} p\left(x_{t+1}, r_{t} \mid x_{1: t}\right)=\sum_{r_{t}=0}^{t} p\left(r_{t} \mid x_{1: t}\right) p\left(x_{t+1} \mid x_{1: t}, r_{t}\right) \\
& =\sum_{r_{t}=0}^{t} p\left(r_{t} \mid x_{1: t}\right) p\left(x_{t+1} \mid x_{\left(t-r_{t}+1\right): t}\right)=\sum_{r_{t}=0}^{t} p\left(r_{t} \mid x_{1: t}\right) p\left(x_{t+1} \mid x^{\left(r_{t}\right)}\right) .
\end{aligned}
$$

6 